\title{
Short- and Medium-Term Induced Ionization in the Earth Atmosphere by Galactic and Solar Cosmic Rays
}

\author{
Alexander Mishev ${ }^{1,2}$ \\ ${ }^{1}$ Institute for Nuclear Research and Nuclear Energy, Bulgarian Academy of Sciences, 1784 Sofia, Bulgaria \\ ${ }^{2}$ Sodankyla Geophysical Observatory (Oulu Unit), University of Oulu, 90014 Oulu, Finland \\ Correspondence should be addressed to Alexander Mishev; alex_mishev@yahoo.com
}

Received 24 August 2012; Accepted 24 October 2012

Academic Editor: Prodromos Zanis

Copyright (C) 2013 Alexander Mishev. This is an open access article distributed under the Creative Commons Attribution License, which permits unrestricted use, distribution, and reproduction in any medium, provided the original work is properly cited.

\begin{abstract}
The galactic cosmic rays are the main source of ionization in the troposphere of the Earth. Solar energetic particles of $\mathrm{MeV}$ energies cause an excess of ionization in the atmosphere, specifically over polar caps. The ionization effect during the major ground level enhancement 69 on January 20, 2005 is studied at various time scales. The estimation of ion rate is based on a recent numerical model for cosmic-ray-induced ionization. The ionization effect in the Earth atmosphere is obtained on the basis of solar proton energy spectra, reconstructed from GOES 11 measurements and subsequent full Monte Carlo simulation of cosmic-ray-induced atmospheric cascade. The evolution of atmospheric cascade is performed with CORSIKA 6.990 code using FLUKA 2011 and QGSJET II hadron interaction models. The atmospheric ion rate is explicitly obtained for various latitudes, namely, $40^{\circ} \mathrm{N}, 60^{\circ} \mathrm{N}$ and $80^{\circ} \mathrm{N}$. The time evolution of obtained ion rates is presented. The short- and medium-term ionization effect is compared with the average effect due to galactic cosmic rays. It is demonstrated that ionization effect is significant only in subpolar and polar atmosphere during the major ground level enhancement of January 20, 2005. It is negative in troposphere at midlatitude, because of the accompanying Forbush effect.
\end{abstract}

\section{Introduction}

Cosmic rays are high, ultrahigh, and extremely high energy particles of extraterrestrial origin, mostly protons. Cosmic rays (CRs) constantly impinge the Earth's atmosphere. While the low-energy particles are absorbed in the atmosphere, those with energies greater than $1 \mathrm{GeV} /$ nucleon generate new particles through interactions with atomic nuclei. They are an important source of ionization in the Earth atmosphere [1]. The ionization in the stratosphere and troposphere is governed by galactic cosmic rays [2]. They initiate a complicated nuclear-electromagnetic-muon cascade resulting in an ionization of the ambient air. In such a cascade a small fraction of the initial primary particle energy reaches the ground as high energy secondary particles. Most of the primary energy is released in the atmosphere by ionization and excitation of the air molecules, resulting in an ionization of the ambient air. The maximum in secondary particle energy release is observed at altitudes of $15-26 \mathrm{~km}$ depending on latitude and solar activity level. This is the Pfotzer maximum.
The galactic cosmic ray (GCR) is affected by solar activity. They follow the 11-year solar cycle and respond to long and short time scale solar-wind variations. They are modulated with the opposite phase, that is, the higher solar activity, the lower the intensity of galactic CRs is. Solar energetic particles (SEPs) are accelerated during explosive energy release on the Sun and by acceleration processes in the interplanetary space. They enter the atmosphere sporadically, with a greater probability during periods of increased solar activity. In addition the heliosphere transient phenomena lead to a strong, relatively short suppression of GCR intensity in the vicinity of Earth, followed by a slower recovery on the time scale of several days known as Forbush decrease [3]. These events are generally interpreted as a result of the influence of coronal mass ejections (CMEs) and/or high-speed streams of the solar wind from the coronal holes on the background CRs.

The abundances of CR are nearly independent of the energy. For lower energies below $1 \mathrm{GeV} /$ nucleon, the relative abundance of heavier nuclei increases, particularly around 
solar maximum, because they are less modulated than protons. In addition for a given energy, protons produce an atmospheric cascade that develops deeper in the atmosphere than cascades from heavier nuclei.

The investigation of ionization processes in atmosphere is important for better understanding of various processes and space weather mechanisms [1]. As example galactic cosmic rays influence via ionization the electrical parameters of planetary atmospheres. They are also related to atmospheric chemistry, as example the ozone depletion in the stratosphere. In addition to CR particles, solar electromagnetic X and UV radiations can affect the ionosphere and atmosphere, specifically the upper atmosphere. The effect varies geographically following the insolation pattern. It is beyond the topic of this study.

In the work presented here the ionization rate in the atmosphere during the major solar energetic particle event on January 20, 2005 is estimated. The obtained ion rates are compared with the average GCR ion production. This event is considered for study, because it is among the largest solar energetic particles events. In addition, the event occurred during the winter period; therefore the contribution of solar UV radiation could be neglected at polar latitudes for further atmospheric studies.

\section{Modeling of CR Induced Atmospheric Ionization}

The estimation of cosmic-ray-induced ionization, as was recently demonstrated, could be performed on the basis of a full Monte Carlo simulation of the atmospheric cascade [4]. At present, with the development of numerical methods, evolution of the knowledge of high energy interactions and nuclear processes, an essential progress in models for cosmicray-induced ionization in the Earth atmosphere is carried out [5-8]. As was recently demonstrated the models agree with $10-20 \%$, the difference is mainly due to the various hadron generators and atmospheric models [2].

The full Monte Carlo simulation of the atmospheric cascade permits to follow the longitudinal cascade evolution in the atmosphere and obtains the energy deposit by different atmospheric cascade components from ground level till the upper atmosphere. These full target models apply the formalism of ionization yield function $Y$

$$
Y(x, E)=\Delta E(x, E) \frac{1}{\Delta x} \cdot \frac{1}{E_{\text {ion }}} \cdot \Omega,
$$

where $\Delta E$ is the deposited energy in layer $\Delta x$ in the atmosphere and $\Omega$ is a geometry factor, integration over solid angle, $E_{\text {ion }}=35 \mathrm{eV}$, which is the energy necessary for production of one ion pair $[9,10]$. The ionization yield function represents the number of ion pairs produced at given altitude $x$ in the atmosphere by given primary cosmic ray nuclei with kinetic energy $E$ on the top of the atmosphere. In fact the ionization yield function represents the ionization capacity in air by given primary CR particle.
The estimation of atmospheric ion rate production is obtained by convolution of $Y(x, E)$ and SEP spectrum as shown in

$$
q\left(h, \lambda_{m}\right)=\int_{E_{0}}^{\infty} D\left(E, \lambda_{m}\right) Y(h, E) \rho(h) d E,
$$

where $D\left(E, \lambda_{m}\right)$ is the differential primary cosmic ray spectrum at given geomagnetic latitude $\lambda_{m}$ for a given component of primary cosmic ray, $Y$ is the yield function (1), and $\rho(h)$ is the atmospheric density $\left(\mathrm{g} \cdot \mathrm{cm}^{-3}\right)$. We express $x$, during the simulations in $\mathrm{g} \cdot \mathrm{cm}^{-2}$, which is a residual atmospheric depth, that is, the amount of matter (air) overburden above a given altitude in the atmosphere. This is naturally related to the development of the cascade. Subsequently the mass overburden is transformed as altitude above sea level (a.s.l.) in $[\mathrm{km}]$.

In this study the simulation of the development of atmospheric cascade is carried out with CORSIKA 6.990 code [11] with corresponding hadron interaction generators FLUKA 2011 [12] and Quark Gluon String with JETs QGSJET II [13]. COsmic Ray SImulations for KASKADE (CORSIKA) code is the most widely used atmospheric cascade simulation tool. The code simulates the interactions and decays of various nuclei, hadrons, muons, electrons, and photons in the atmosphere. The particles are tracked through the atmosphere until they undergo reactions with an air nucleus or in the case of unstable secondary particles, they decay. The result of the simulations is detailed information about the type, energy, momenta, location, and arrival time of the produced secondary particles at given selected altitude a.s.l. The primary particles that can be considered are protons, light, middle, and heavy nuclei up to iron.

\section{Ionization Effect in the Atmosphere due to Galactic and SEP}

The described above formalism is applicable for the whole atmosphere where CR could affect from ground level to altitude of $100 \mathrm{~km}$ a.s.l. After obtaining the energy deposition from all secondary cosmic ray particles, respectively, ionization yield function $Y$ (1) on the basis of (2), the ion rate as ion pairs per second in $\mathrm{cm}^{3}$ is obtained. The ion rates are presented as ion pairs per second, per $\mathrm{cm}^{3}$, and per atmosphere.

3.1. Ion Rates at Solar Minimum and Maximum at Various Atmospheric Depths. The cosmic-ray-induced ionization by GCR at solar minimum and maximum is obtained for ground level to the upper atmosphere. In Figure 1 the obtained ion rates are presented for various atmospheric depths, namely, $100 \mathrm{~g} \mathrm{~cm}^{-2}$ (16 $\mathrm{km}$ a.s.1.), $300 \mathrm{~g} \cdot \mathrm{cm}^{-2}$ (10 $\mathrm{km}$ a.s.l.), $500 \mathrm{~g} \cdot \mathrm{cm}^{-2}$ (5 $\mathrm{km}$ a.s.1.), and $700 \mathrm{~g} \cdot \mathrm{cm}^{-2}(3 \mathrm{~km}$ a.s.l.) as a function of the rigidity cut-off.

It is demonstrated that solar activity affects ion rate production significantly at high latitudes, while at the Equator (15 GV cut-off), this effect is not so strong. Such computation of GCR-induced ionization permits to estimate the ion rate production at various time scales. Therefore the average ion 


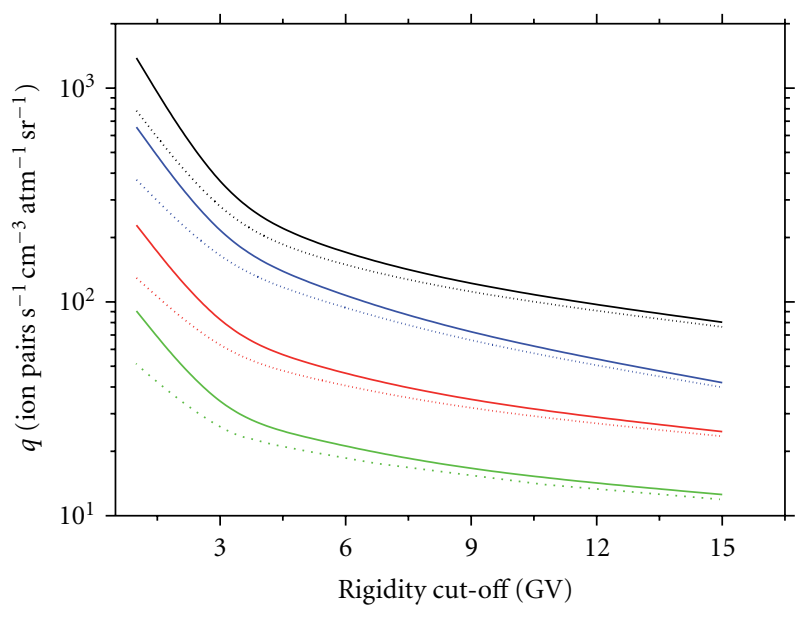

Solar minimum, solar maximum

$100 \mathrm{~g} \mathrm{~cm}^{-2} \quad \ldots .$.

$300 \mathrm{~g} \mathrm{~cm}^{-2}$

$500 \mathrm{~g} \mathrm{~cm}^{-2}$

$700 \mathrm{~g} \mathrm{~cm}^{-2}$

FIGURE 1: Cosmic-ray-induced ionization by GCR at solar minimum and maximum at various atmospheric depths.

rate due to GCR could be estimated for specific conditions (solar minimum and maximum). Hence, the obtained ion rates due to GCR permit to compare the SEP ionization effect with average ionization $\mathrm{CR}$ effect in a realistic manner.

3.2. Ionization Effect on January 20, 2005, during the Major GLE 69. In addition to continuous ionization in the Earth's atmosphere caused by galactic cosmic ray a sporadic ionization occurred during solar energetic particle events, potentially affecting the Earth's environment [14]. In general such events are low energy and are not able to initiate atmospheric cascades. Their ionization effect is limited to the upper polar atmosphere. Hence the studies of the effects caused by solar energetic particles are usually limited to the upper atmosphere above $30 \mathrm{~km}$. However, it was recently demonstrated [15-18] that during some major ground level enhancements (GLEs), which are characterized by very high energy of solar particles, the ionization effect is important specifically over polar atmosphere.

On January 20, 2005 all energy channels of GOES satellite simultaneously register enhancement of proton flux. The (SEP) onset was registered at 6:50 UT, 3 minutes before maximum of X-ray flare. During the first hour the proton spectrum parameters change dramatically [19]. The changes of the spectra could be connected with some particle acceleration mechanism preceding the CME launch. The next 10 hours of acceleration produce spectra with very stable parameters [20], most likely formed at CME driven shock site.

A well-studied event [19] such as ground level enhancement 69 on January 20, 2005 was considered for analysis. Since this event is among the largest (the second largest in observation history), the ionization effect is expected to be maximal. The event on January 20, 2005 is characterized by an anisotropic component with a very hard spectrum at the onset of the event, followed by a long isotropic emission with a softer spectrum [21]. The spectral index for SEP during GLE is typically between 4 and 6 . In this work the solar proton spectrum is obtained on the basis of GOES 11 satellite measurements (high energy channels) and additional data $[22,23]$.

The spectrum of solar protons is expressed in two different moments: at 08:00 UT a high energy part with a slope of 2.32 and at 23:00 UT low energy part with a slope of 3.43. During the simulation, a realistic winter profile of the Earth atmosphere is considered $[24,25]$. It is demonstrated that the ionization effect on event onset at 08:00 UT (Figure 2(a)) is greater than the one produced by delayed component at 23:00 UT (Figure 2(b)). Because the event on January 20, 2005 occurred during the recovery phase of the Forbush decrease and in the following days an additional suppression of the cosmic ray intensity was observed leading to a complicated time profile of cosmic ray flux, the net ionization effect is calculated as a superposition of ion rate from solar energetic particles and from reduced galactic cosmic rays.

In the case of $40^{\circ} \mathrm{N}$ latitude the effect at 08:00 UT is comparable to the average of GCR (Figures 1 and 2(a)). However, the ion rates quickly decrease with altitude (below $5 \mathrm{~km}$ a.s.l.). The ionization effect due to low energy component of the SEP spectrum, namely, at 23:00 UT is negligible (Figure 2(b)). In this case the ion rates are due mostly from reduced GCR.

The situation is quite different for latitude of $60^{\circ} \mathrm{N}$ (Figure 3). The ion rate due to SEP at 08:00 UT is significant. The ion rates from solar particles are greater than ion rates from GCR roughly an order of magnitude. The ionization is significant at altitudes above some $12 \mathrm{~km}$ a.s.l. and decreases in the troposphere. The ion rate at 23:00 UT due to a low energy component, as in a previous case, is negligible. In the case of $80^{\circ} \mathrm{N}$ latitude both components, hard at 08:00 UT, respectively, soft at 23:00 UT cause a significant excess on ion rates in the atmosphere. The effect at 08:00 UT is due mainly to solar protons (Figure 4(a)). It is significant at altitudes above $10-12 \mathrm{~km}$ a.s.l. Because the hard spectrum is with a slope of 2.32, it causes significant ionization even in a low atmosphere. At 23:00 UT the effect due to solar particles is significant at altitudes above $12 \mathrm{~km}$ a.s.l. and decreases in the troposphere, because of the softer spectrum. The ionization effect in a low troposphere is due to a reduced GCR (Figure 4(b)). Computed ion rates permit to estimate the net ionization effect at various time scales and to compare it to the average effect due to GCR.

3.3. Short and Medium Time Ionization Effect due to GLE 69 on January 20, 2005. The ionization effect is maximal during the first $24 \mathrm{~h}$ from the event onset $[14,16,17]$. The $24 \mathrm{~h}$ effect is computed as superposition of SEP and GCR ionization. Since the event occurred during the recovery phase of a strong Forbush decrease, a reduced GCR flux is considered. The $48 \mathrm{~h}$ effect is superposition of $24 \mathrm{~h}$ effect and reduced GCR ionization effect for the following $24 \mathrm{~h}$. The weekly effect is computed taking into account the $24 \mathrm{~h}$ ionization effect from solar protons and complicated GCR flux, considering explicitly the transient phenomena during the week. 


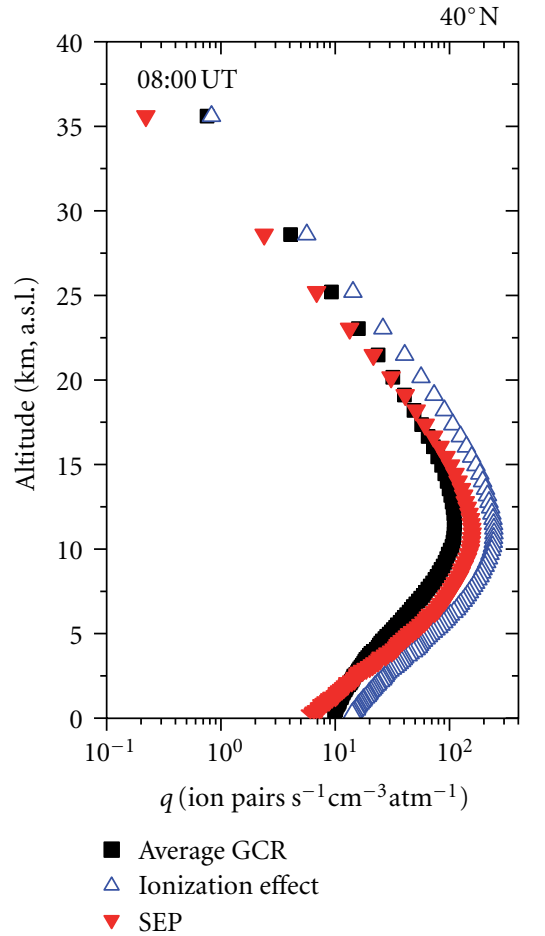

(a)

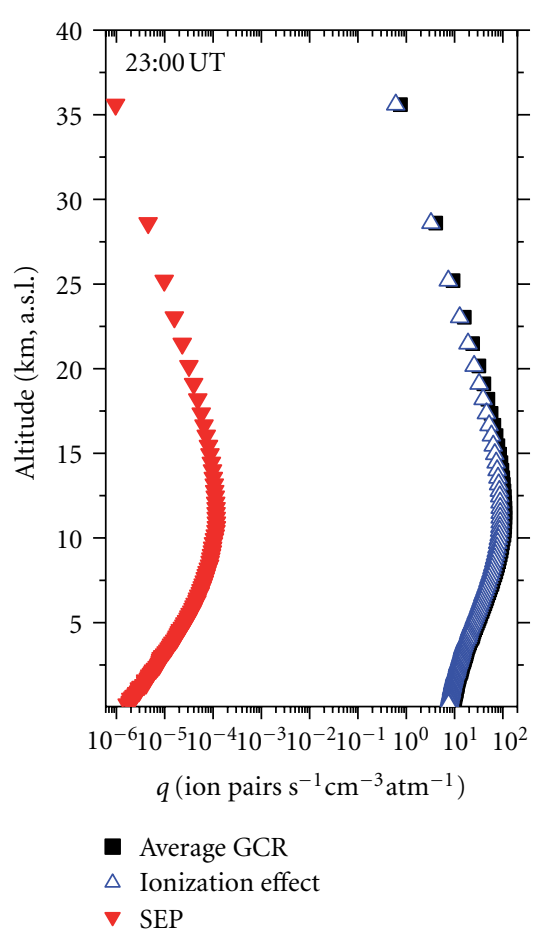

(b)

FIGURE 2: Ionization effect at $40^{\circ} \mathrm{N}$ due to GCR and SCR during GLE 69 on January 20, 2005.

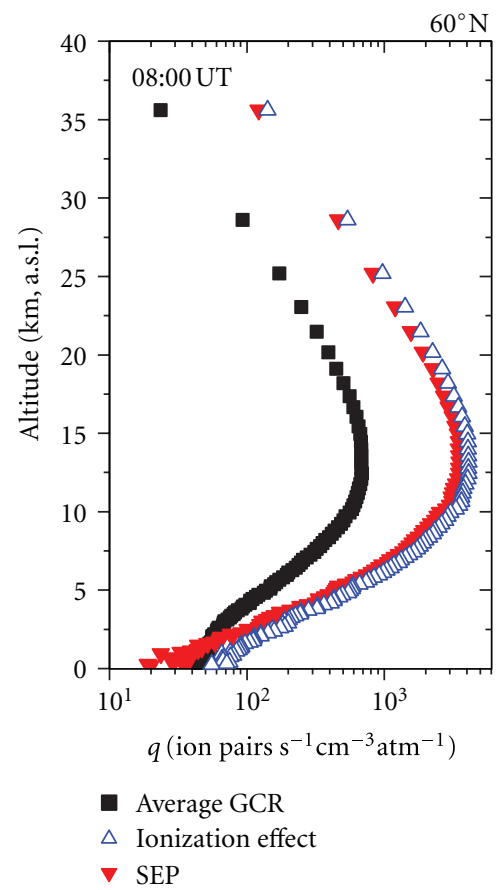

(a)

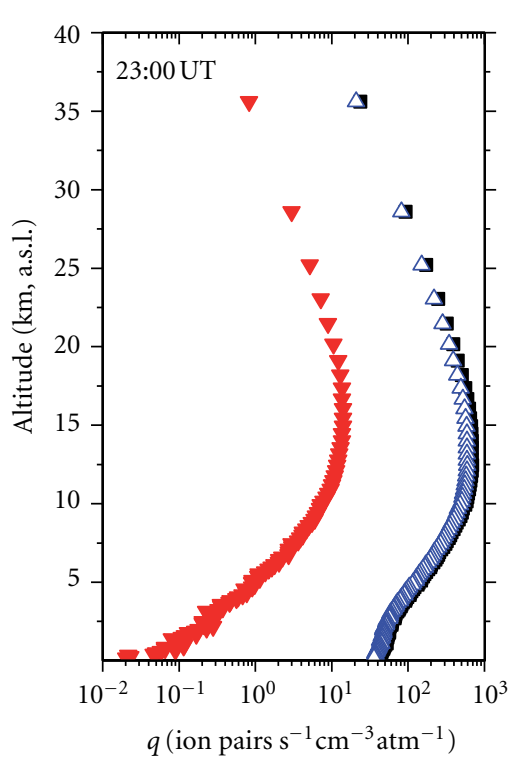

- Average GCR

$\triangle$ Ionization effect

$\nabla$ SEP

(b)

FIgURE 3: Ionization effect at $60^{\circ} \mathrm{N}$ due to GCR and SCR during GLE 69 on January 20, 2005. 


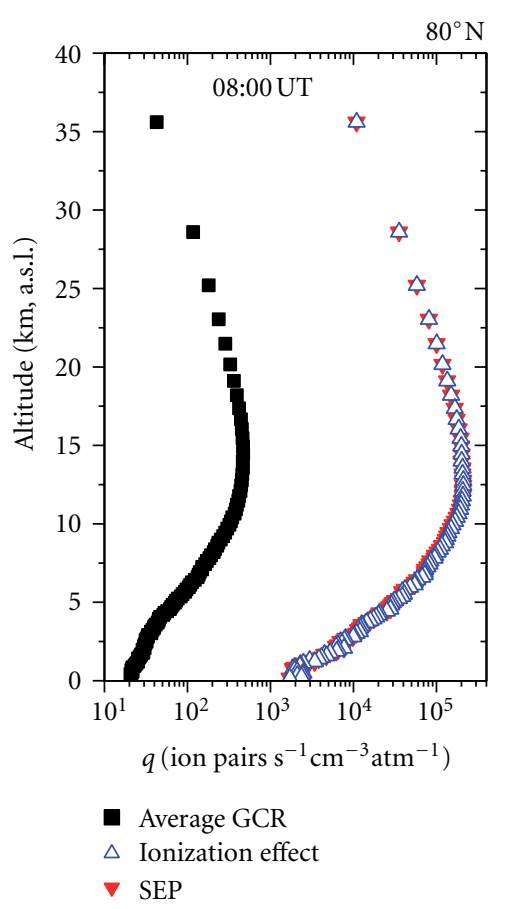

(a)

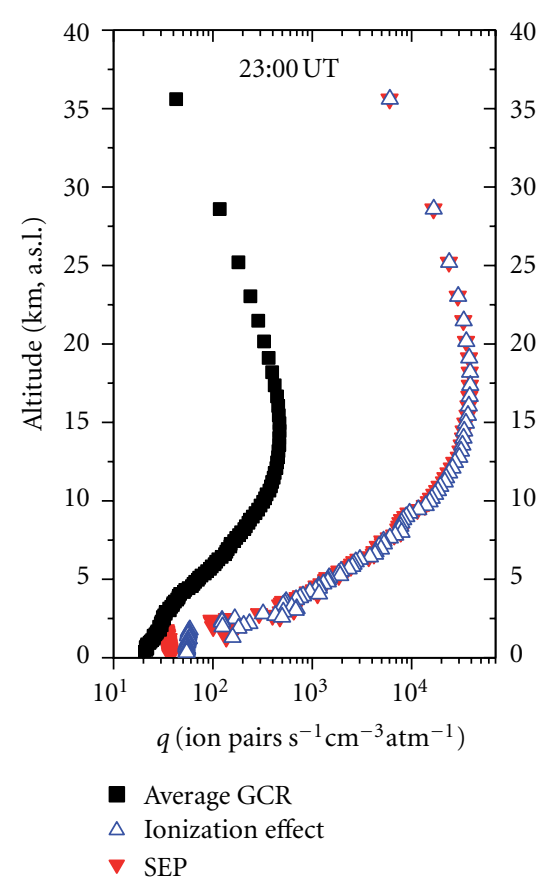

(b)

FIGURE 4: Ionization effect at $80^{\circ} \mathrm{N}$ due to GCR and SCR during GLE 69 on January 20, 2005.

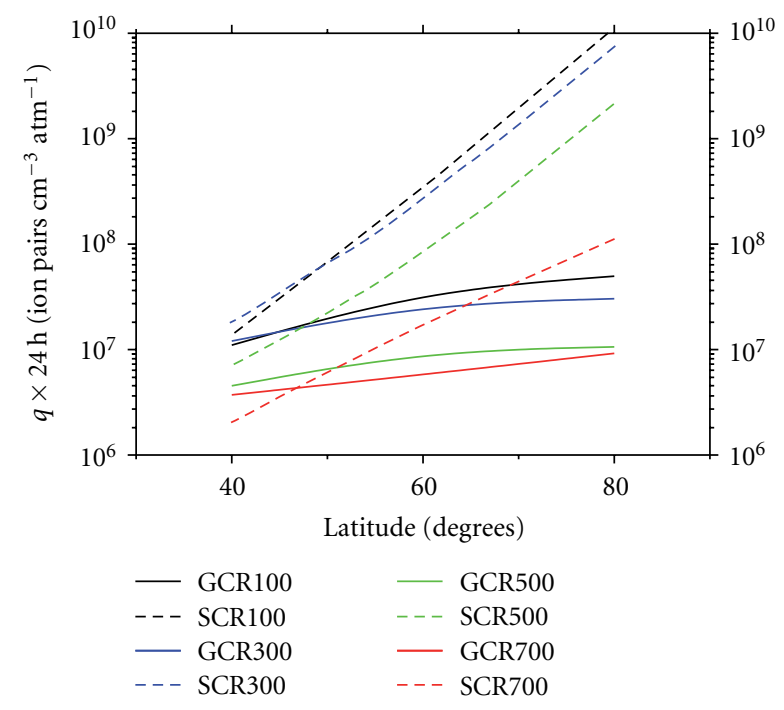

FIgURE 5: $24 \mathrm{~h}$ ionization effect due to solar protons during GLE69, compared with average GCR, as a function of latitude for various observation depths.

The ionization effect at various timescales is computed at various altitudes a.s.l., namely, at $100 \mathrm{~g} \mathrm{~cm}^{-2}$ (16 $\mathrm{km}$ a.s.l.), $300 \mathrm{~g} \cdot \mathrm{cm}^{-2}$ (10 $\mathrm{km}$ a.s.l.), $500 \mathrm{~g} \cdot \mathrm{cm}^{-2}$ (5 $\mathrm{km}$ a.s.l.), and $700 \mathrm{~g} \cdot \mathrm{cm}^{-2}$ ( $3 \mathrm{~km}$ a.s.l.) as a function of the rigidity cut-off. This permits to estimate with good precision in which region of the atmosphere the ionization effect is significant, weak or negative. The Figures 5-7 demonstrate the net ionization

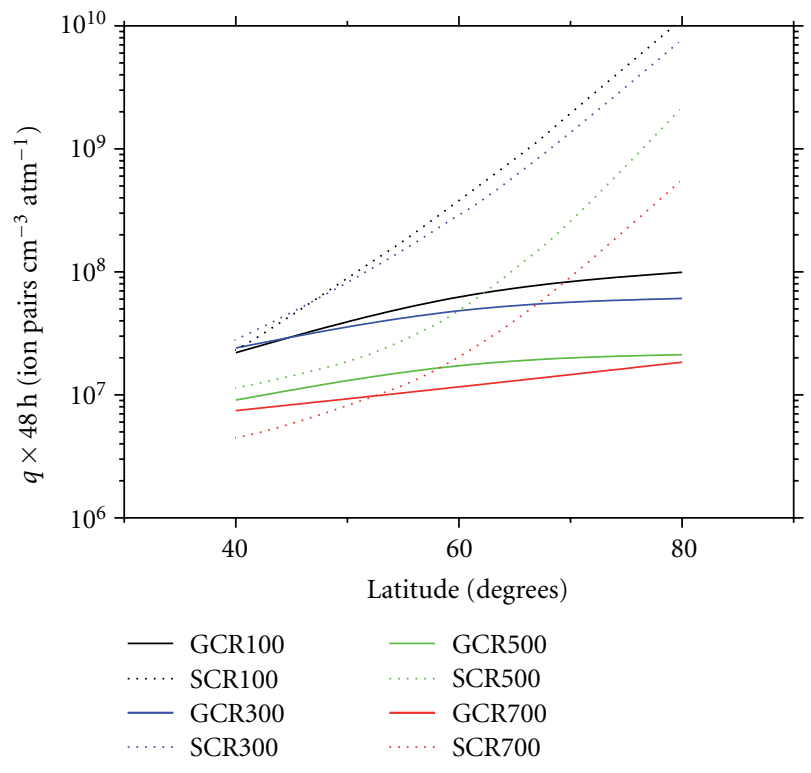

FIGURE 6: $48 \mathrm{~h}$ ionization effect due to solar protons during GLE69, compared with average GCR, as a function of latitude for various observation depths.

effect as a function of the rigidity cut-off at various altitudes compared with average ionization effect from GCR. The average ionization effect from GCR is computed for period corresponding to the week before GLE 69 onset.

In general, as was shown in (Figure 2), the effect at $40^{\circ} \mathrm{N}$ is weak, with excess in the region of Pfotzer maximum. 


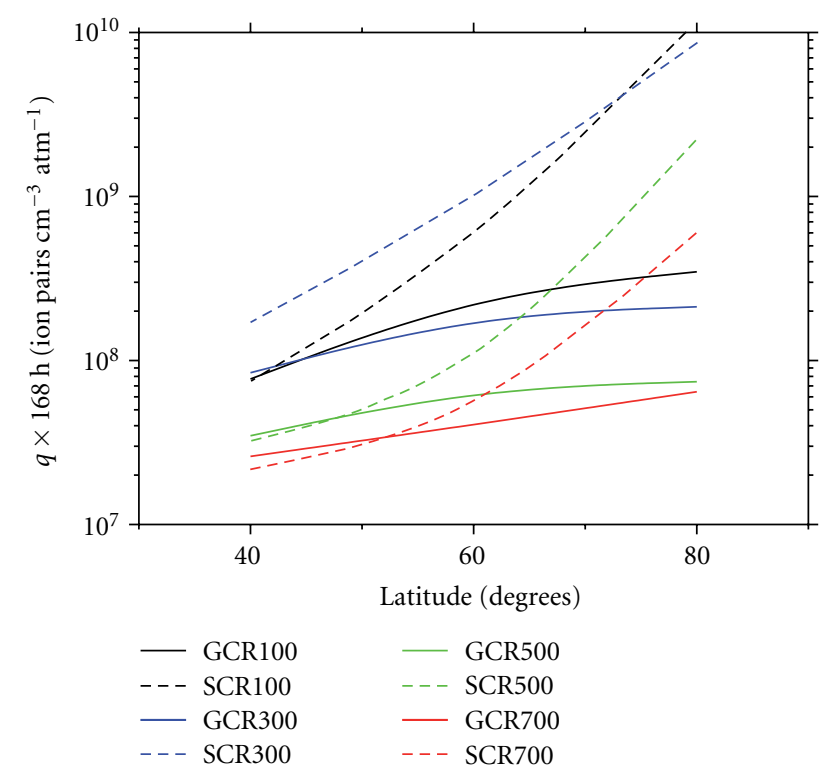

FIGURE 7: Weekly ionization effect due to solar protons during GLE69, compared with average GCR, as a function of latitude for various observation depths.

Therefore the $24 \mathrm{~h}$ is weak in a whole midlatitude atmosphere (Figure 5). We observe negative ionization effect compared to average effect from GCR at $700 \mathrm{~g} \cdot \mathrm{cm}^{-2}$ (red lines on Figure 5). The effect from SEP is weak at $500 \mathrm{~g} \cdot \mathrm{cm}^{-2}$ (green lines). Only at altitudes around some $10 \mathrm{~km}$ a.s.l. the ionization effect due to solar protons is greater than the average due to GCR at $40^{\circ} \mathrm{N}$ latitude. As was expected the effect increases for low rigidity cut-offs, that is, in subpolar and polar regions. We conclude that the $24 \mathrm{~h}$ ionization effect is important in the subpolar and polar atmosphere during major ground level enhancement of January 20,2005, specifically at high altitudes (Figure 5-black and blue dashed lines).

The $48 \mathrm{~h}$ ion production at $40^{\circ} \mathrm{N}$ is comparable with the average due to GCR at altitudes above $500 \mathrm{~g} \cdot \mathrm{cm}^{-2}$ (Figure 6). The $48 \mathrm{~h}$ ion production is below the average due to GCR at altitude of $3 \mathrm{~km}$ for $40^{\circ} \mathrm{N}$. Because the significant ion production at $60^{\circ} \mathrm{N}$ and $80^{\circ} \mathrm{N}$; the $48 \mathrm{~h}$ ionization effect is still strong at these regions, specifically at high altitudes. The $48 \mathrm{~h}$ effect results on $50 \%$ increase compared to the average due to GCR at $60^{\circ} \mathrm{N}$. The effect is even stronger at $80^{\circ} \mathrm{N}$. Therefore the $48 \mathrm{~h}$ ionization effect is important only in the subpolar and polar atmosphere during major ground level enhancement of January 20, 2005 at high altitudes.

The weekly effect at $40^{\circ} \mathrm{N}$ (Figure 7) is clearly negative at low altitude (green and red lines at Figure 7). It is comparable with average GCR (black lines at Figure 7) effect at high altitude. However, the significant ion production during the event onset results on important ionization effect in the region of the Pfotzer maximum at $40^{\circ} \mathrm{N}$ (blue lines at Figure 7). The weekly effect is weak at the troposphere even at subpolar latitudes. However at $80^{\circ} \mathrm{N}$ the weekly effect is still important.

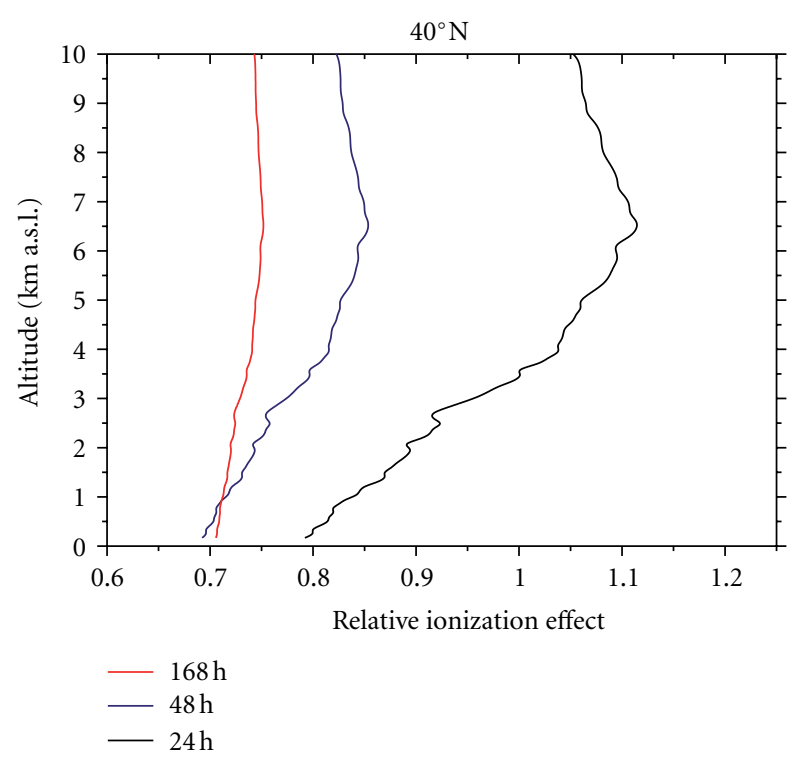

FIGURE 8: Relative to the average GCR ionization effect for $24 \mathrm{~h}, 48 \mathrm{~h}$, and $168 \mathrm{~h}$ at $40^{\circ} \mathrm{N}$ in troposphere.

An illustration of the above conclusions is shown in Figure 8. Figure 8 demonstrates the relativity to the average GCR ionization effect for $24 \mathrm{~h}, 48 \mathrm{~h}$, and $168 \mathrm{~h}$ at $40^{\circ} \mathrm{N}$ in the low troposphere. It is shown that the $24 \mathrm{~h}$ ionization effect is positive in a tight region of the troposphere above some $5 \mathrm{~km}$ a.s.l. In this region the interplay between reduced GCR flux and SEP leads to slight increase of the ionization. As was expected the $48 \mathrm{~h}$ is negative, as well as the weekly ionization effect (red and blue lines). In the upper troposphere the $24 \mathrm{~h}$ ionization effect is positive compared to the average of GCR only is a small region (Figure 9). The ionization effect is negative in the stratosphere. The $48 \mathrm{~h}$ ionization effect is clearly negative as was mentioned above.

Similarly it is demonstrated that 24 ionization effect at $60^{\circ} \mathrm{N}$ is not important in the lower troposphere (Figure 10). The $48 \mathrm{~h}$ ionization effect is not important as well and is slightly negative in planetary boundary layer.

Taking into account the time evolution of the obtained ion rates, the conclusion is the ionization effect is nearly negative for $40^{\circ} \mathrm{N}$, especially in the troposphere and small for $60^{\circ} \mathrm{N}$, because of the accompanying Forbush decrease during the event. The ionization effect is important only in the subpolar and polar atmosphere during major ground level enhancement of January 20, 2005 at high altitudes.

\section{Applications and Discussion}

The application of recent Monte Carlo CR ionization models is related to the study of various processes in the atmosphere, specifically related to atmospheric chemistry and physics [26, 27]. The influence of GCR should not be neglected in investigations of the tropospheric and stratospheric chemistry and dynamics. The CR ionization models show that the effects of galactic cosmic rays on the atmosphere are statistically 


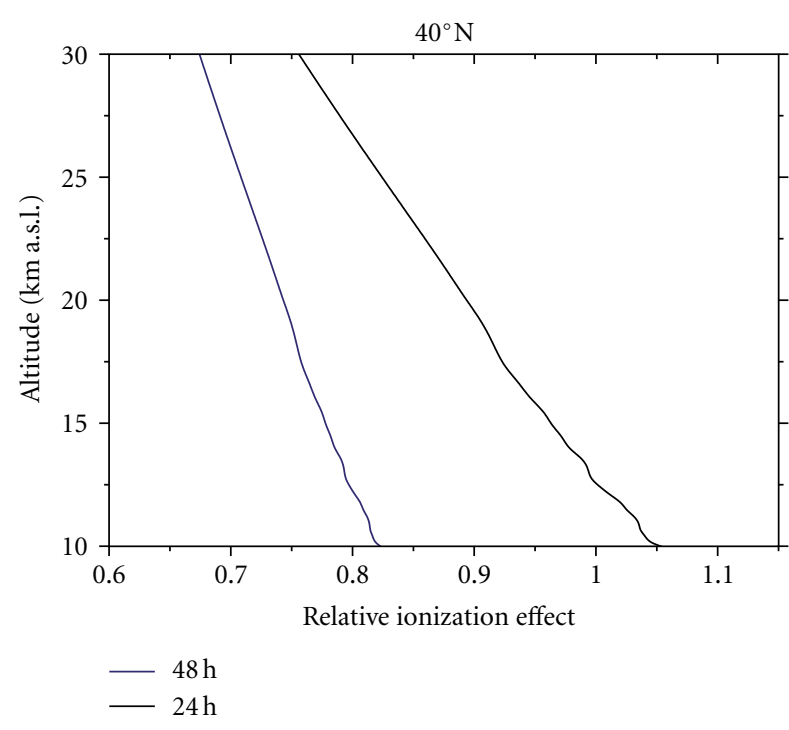

FIgURE 9: Relative to the average GCR ionization effect for $24 \mathrm{~h}$ and $48 \mathrm{~h}$ at $40^{\circ} \mathrm{N}$ in the upper troposphere and stratosphere.
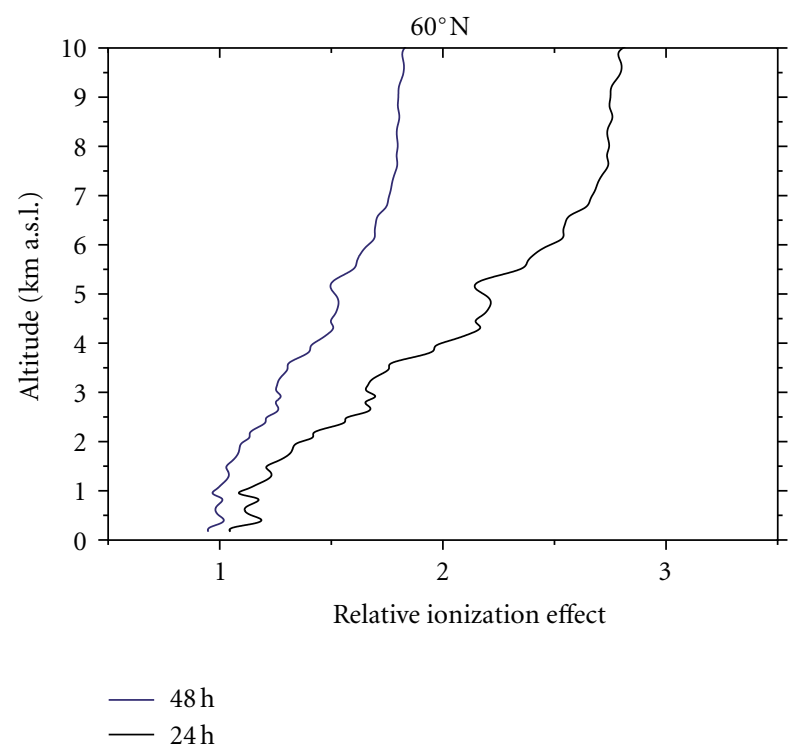

FIGURE 10: Relative to the average GCR ionization effect for $24 \mathrm{~h}$ and $48 \mathrm{~h}$ at $60^{\circ} \mathrm{N}$ in the troposphere.

significant in large geographic regions and for a number of relevant atmospheric species.

It was recently demonstrated for Southern hemispheric troposphere that $\mathrm{NO}_{x}$ increases more than $20 \%$ in the polar region [26]; $\mathrm{HO}_{x}$ decreases of $3 \%$ in the midlatitude upper troposphere; $\mathrm{HNO}_{3}$ increases by more than $10 \%$ between the South Pole and subtropics and $\mathrm{O}_{3}$ increases by up to 3\% throughout the troposphere to $20 \mathrm{~km}$ between the South Pole to $20^{\circ} \mathrm{N}$. Respectively, for Northern hemispheric troposphere $\mathrm{HNO}_{3}$ marginally significant increases in the midlatitude upper troposphere and $\mathrm{O}_{3}$ significant decreases in the polar upper troposphere. Undoubtedly these effects are related with the planetary distribution of GCR.
In addition it was demonstrated that major solar events leading to GLEs could also impact the Earth atmosphere [28]. According to the current knowledge, the cosmic-ray-induced ionization of the atmosphere, due to solar and galactic cosmic rays produces additional chemical sources and influences ozone molecules in the atmosphere [29] via photochemistry [30]. Recently, was demonstrated, that the ionization of the polar atmosphere by SEPs is responsible for the impact on neutral constituents. In addition it was shown that even events with limited particle flux could change the abundance of the minor constituents in the mesosphere and the upper stratosphere [31].

The obtained results presented here are related to these studies, since the atmospheric ionization is related to the mentioned above phenomena. Recent studies at the upper atmosphere on short-medium time [32, 33] demonstrate that the January 2005 SPEs caused large enhancements in the northern polar mesospheric $\mathrm{HO}_{x}$ and $\mathrm{NO}_{x}$ constituents (both observed and modeled). Observations indicated large mesospheric increases in $\mathrm{OH}$ (up to 4 ppbv) and $\mathrm{HO}_{2}$ $(>0.5 \mathrm{ppbv})$ as a result of the SPEs during the time period 16-21 January in the 60-85N latitude. The WACCM3 simulations showed quantitatively similar, although somewhat larger enhancements in $\mathrm{OH}$ and $\mathrm{HO}_{2}$. These large $\mathrm{HO}_{x}$ enhancements led to considerable ozone decreases of greater than $40 \%$ throughout most of the northern polar mesosphere during the SPE period. All these studies demonstrate that SEP impact on the atmosphere is more important than GLE particles in the upper atmosphere. However, for better understanding of the atmospheric chemistry and physics process is the investigation if their impact is important.

While the recent model studies of cosmic-ray-induced ionization during the major GLE 69 on January 20, 2005 focus on the onset of the event and the next following hours, when the effect is maximal, in the study presented here we focus on short- to mid-term ionization effect. Previous works did not concern the mid-term ionization effect in details for various altitudes in the atmosphere. In addition several of the previous studies are limited to a high atmosphere [32] or only on the contribution of heavy ions [34]. Therefore the results presented here extend the recent studies of such events and give basis for further improvement of model studies related to atmospheric physics phenomena.

\section{Conclusion}

The application of Monte Carlo methods for investigation of cosmic-ray-induced ionization is very important, because it is possible to consider explicitly the hadron component. It gives the possibility to estimate middle and low atmospheric effects related to cosmic-ray-induced ionization realistically. As was recently demonstrated their application in a specific conditions $[15,17]$ permits detailed study of the ionization effect, specifically at middle and low altitudes [16].

The effect of sporadic solar energetic particle events is limited on a global scale but most energetic events could be strong locally, specifically in subpolar and polar regions, affecting the physical-chemical properties of the upper atmosphere. Since the large SEP events leading to GLEs are 
different on spectra and composition, their detailed study is connected with detailed information about heliospheric and geospace conditions. In this connection extension of the existing models to the upper atmosphere is very important, as well as their comparison with analytical models.

The ionization effects in the upper atmosphere during GLE 69 on January 20, 2005 are well studied [32, 33]. In this study it is shown that ionization effect is significant at subpolar and polar atmosphere, with fast tropospheric decrease $[16,17]$. Previous studies are focused on a maximal phase of ionization effect during the first hours from GLE onset. In this study the obtained ion rates are used to estimate the mid-term ionization effect. It is demonstrated that the ionization effect is nearly negative compared to GCR at middle latitudes for short- to mid-term periods. This is very important for further studies of the influence of GLE particles on atmospheric chemistry and physics.

It has a general agreement that cosmic-ray-induced ionization of the atmosphere, due to solar and galactic cosmic rays, produces additional chemical sources and influences ozone molecules in the atmosphere. As was mentioned above the ionization effect due to SEPs is important only in a subpolar and polar atmosphere. This is very important for recent composition changes studies. The January 20, 2005 SPEs caused large enhancements in the northern polar $\mathrm{HO}_{x}$ and $\mathrm{NO}_{x}$ constituents in mesosphere, which lead to ozone decrease in the order of $40 \%$. However on the basis of the obtained ion rate it is demonstrated that the tropospheric and stratospheric ionization effects due to SEPs are important on short to medium time scales in polar atmosphere and are nearly negative compared to the average GCR on medium time scales at middle latitudes (because of the accompanying Forbush decrease). More detailed studies of the influence on minor components of the atmosphere deserve special interest.

In addition, it was recently demonstrated [34] that the contribution of light nuclei, specifically Helium could be important for total ionization even at middle latitudes during GLE 69. The extension of such models, specifically in the upper atmosphere on the basis of a full Monte Carlo simulation of the atmospheric cascade with combination with analytical procedures [35] will contribute to deep understanding of the impact of solar and galactic cosmic rays on the atmosphere of the Earth and their connection to the space weather [36-38] and atmospheric physics [39].

\section{Acknowledgments}

The author warmly acknowledges the high energy Division of the Institute for Nuclear Research and Nuclear Energy of Bulgarian Academy of Sciences, namely, V. Guenchev, S. Piperov, E. Puncheva, and P. Konstantitov for the given computational time.

\section{References}

[1] G. A. Bazilevskaya, I. G. Usoskin, E. O. Flückiger et al., "Cosmic ray induced ion production in the atmosphere," Space Science Reviews, vol. 137, no. 1-4, pp. 149-173, 2008.
[2] I. G. Usoskin, L. Desorgher, P. Velinov et al., "Ionization of the earth's atmosphere by solar and galactic cosmic rays," Acta Geophysica, vol. 57, no. 1, pp. 88-101, 2009.

[3] S. E. Forbush, "Cosmic-ray intensity variations during two solar cycles," Journal of Geophysical Research, vol. 63, no. 4, pp. 651-669, 1958.

[4] I. G. Usoskin, O. G. Gladysheva, and G. A. Kovaltsov, "Cosmic ray-induced ionization in the atmosphere: spatial and temporal changes," Journal of Atmospheric and Solar-Terrestrial Physics, vol. 66, no. 18, pp. 1791-1796, 2004.

[5] L. Desorgher, E. O. Flückiger, M. Gurtner, M. R. Moser, and R. Bütikofer, "Atmocosmics: a geant 4 code for computing the interaction of cosmic rays with the earth's atmosphere," International Journal of Modern Physics A, vol. 20, no. 29, pp. 6802-6804, 2005.

[6] I. G. Usoskin and G. A. Kovaltsov, "Cosmic ray induced ionization in the atmosphere: full modeling and practical applications," Journal of Geophysical Research D, vol. 111, Article ID D21206, 2006.

[7] A. Mishev and P. I. Y. Velinov, "Atmosphere ionization due to cosmic ray protons estimated with Corsika code simulations," Comptes Rendus de L'Academie Bulgare des Sciences, vol. 60, no. 3, pp. 225-230, 2007.

[8] P. I. Y. Velinov, A. Mishev, and L. Mateev, "Model for induced ionization by galactic cosmic rays in the Earth atmosphere and ionosphere," Advances in Space Research, vol. 44, no. 9, pp. 1002-1007, 2009.

[9] P. I. Y. Velinov, G. Nestorov, and L. Dorman, Cosmic Ray Influence on the Ionosphere and on the Radio-Wave Propagation, Bulgarian Academy of Sciences Publishing House, Sofia, Bulgaria, 1974.

[10] H. S. Porter, C. H. Jackman, and A. E. S. Green, "Efficiencies for production of atomic nitrogen and oxygen by relativistic proton impact in air," The Journal of Chemical Physics, vol. 65, no. 1, pp. 154-167, 1976.

[11] D. Heck, J. Knapp, J. N. Capdevielle et al., "CORSIKA: a monte carlo code to simulate extensive air showers," Forschungszentrum Karlsruhe Report FZKA 6019, 1998.

[12] G. Battistoni, S. Muraro, P. R. Sala et al., "The FLUKA code: description and benchmarking," in Proceedings of theHadronic Shower Simulation Workshop, M. Albrow and R. Raja, Eds., vol. 896 of AIP Conference Proceeding, pp. 31-49, September 20062007.

[13] S. Ostapchenko, "QGSJET-II: towards reliable description of very high energy hadronic interactions," Nuclear Physics B, vol. 151, no. 1, pp. 143-146, 2006.

[14] R. Vainio, L. Desorgher, D. Heynderickx et al., "Dynamics of the Earth's particle radiation environment," Space Science Reviews, vol. 147, no. 3-4, pp. 187-231, 2009.

[15] A. Mishev, P. I. Y. Velinov, and L. Mateev, "Atmospheric ionization due to solar cosmic rays from 20 January 2005 calculated with Monte Carlo simulations," Comptes Rendus de L'Academie Bulgare des Sciences, vol. 63, no. 11, pp. 1635-1642, 2010.

[16] I. G. Usoskin, G. A. Kovaltsov, I. A. Mironova, A. J. Tylka, and W. F. Dietrich, "Ionization effect of solar particle GLE events in low and middle atmosphere," Atmospheric Chemistry and Physics, vol. 11, no. 5, pp. 1979-1988, 2011.

[17] A. L. Mishev, P. I. Y. Velinov, L. Mateev, and Y. Tassev, "Ionization effect of solar protons in the Earth atmosphere-case study of the 20 January 2005 SEP event," Advances in Space Research, vol. 48, no. 7, pp. 1232-1237, 2011. 
[18] A. Mishev, P. I. Y. Velinov, and L. Mateev, "Ion production rate profiles in the atmosphere due to solar energetic particles on 28 october 2003 obtained with CORSIKA 6.52 simulations," Comptes Rendus de L'Academie Bulgare des Sciences, vol. 64, no. 6, pp. 859-866, 2011.

[19] R. Butikofer, E. O. Fluckiger, L. Desorgher, and M. R. Moser, "The extreme solar cosmic ray particle event on 20 January 2005 and its influence on the radiation dose rate at aircraft altitude," Science of the Total Environment, vol. 391, no. 2-3, pp. 177-183, 2008.

[20] N. K. Bostanjyan, A. A. Chilingarian, V. S. Eganov, and G. G. Karapetyan, "On the production of highest energy solar protons at 20 January 2005," Advances in Space Research, vol. 39, no. 9, pp. 1456-1459, 2007.

[21] C. Plainaki, A. Belov, E. Eroshenko, H. Mavromichalaki, and V. Yanke, "Modeling ground level enhancements: event of 20 January 2005," Journal of Geophysical Research A, vol. 112, no. 4, Article ID A04102, 2007.

[22] R. A. Mewaldt, M. D. Looper, C. M. S. Cohen et al., "Solarparticle energy spectra during the large events of OctoberNovember 2003 and January 2005," in Proceedings of the 29th International Cosmic Ray Conference, vol. 1, pp. 111-114, Pune, India, 2005.

[23] V. S. Makhmutov, G. A. Bazilevskaya, B. B. Grozdevsky et al., "Solar cosmic ray spectra in the 20 January GLE: comparison of simulations with ballon and neutron monitor observations," in Proceedings of the 31th International Cosmic Ray Conference, pp. 1-4, Lodz, Poland, 2009.

[24] A. Mishev and P. I. Y. Velinov, "Effects of atmospheric profile variations on yield ionization function Y in the atmosphere," Comptes Rendus de L'Academie Bulgare des Sciences, vol. 61, no. 5, pp. 639-644, 2008.

[25] A. L. Mishev and P. Velinov, "The effect of model assumptions on computations of cosmic ray induced ionization in the atmosphere," Journal of Atmospheric and Solar-Terrestrial Physics, vol. 72, no. 5-6, pp. 476-481, 2010.

[26] M. Calisto, I. Usoskin, E. Rozanov, and T. Peter, "Influence of Galactic Cosmic Rays on atmospheric composition and dynamics," Atmospheric Chemistry and Physics, vol. 11, no. 9, pp. 4547-4556, 2011.

[27] L. I. Dorman, Cosmic Rays in the Earth's Atmosphere and Underground, Kluwer Academic, Dordrecht, The Netherlands, 2004.

[28] B. Funke, A. Baumgaertner, M. Calisto et al., "Composition changes after the "halloween" solar proton event: the HighEnergy Particle Precipitation in the Atmosphere (HEPPA) model versus MIPAS data intercomparison study," Atmospheric Chemistry and Physics Discussions, vol. 11, no. 3, pp. 9407-9514, 2011.

[29] A. Krivolutsky, A. Kuminov, and T. Vyushkova, "Ionization of the atmosphere caused by solar protons and its influence on ozonosphere of the Earth during 1994-2003," Journal of Atmospheric and Solar-Terrestrial Physics, vol. 67, no. 1-2, pp. 105-117, 2005

[30] A. A. Krivolutsky, A. V. Klyuchnikova, G. R. Zakharov, T. Y. Vyushkova, and A. A. Kuminov, "Dynamical response of the middle atmosphere to solar proton event of July 2000: threedimensional model simulations," Advances in Space Research, vol. 37, no. 8, pp. 1602-1613, 2006.

[31] A. Damiani, M. Storini, M. Laurenza, and C. Rafanelli, "Solar particle effects on minor components of the Polar atmosphere," Annales Geophysicae, vol. 26, no. 2, pp. 361-370, 2008.
[32] C. H. Jackman, D. R. Marsh, F. M. Vitt et al., "Short- and medium-term atmospheric constituent effects of very large solar proton events," Atmospheric Chemistry and Physics, vol. 8, no. 3, pp. 765-785, 2008.

[33] C. H. Jackman, D. R. Marsh, F. M. Vitt et al., "Northern Hemisphere atmospheric influence of the solar proton events and ground level enhancement in January 2005," Atmospheric Chemistry and Physics, vol. 11, no. 13, pp. 6153-6166, 2011.

[34] A. Mishev, P. I. Y. Velinov, and L. Mateev, "Atmospheric ionization due to solar cosmic rays from 20 January 2005 calculated with Monte Carlo simulations," Comptes Rendus de L'Academie Bulgare des Sciences, vol. 63, no. 11, pp. 1635-1642, 2010.

[35] P. I. Y. Velinov, S. Asenovski, and L. Mateev, "Simulation of cosmic ray ionization profiles in the middle atmosphere and lower ionosphere on account of characteristic energy intervals," Comptes rendus de l'Academie bulgare des Sciences, vol. 64, no. 9, pp. 1303-1310, 2011.

[36] K. Kudela, M. Storini, M. Y. Hofer, and A. Belov, "Cosmic rays in relation to space weather," Space Science Reviews, vol. 93, no. 1-2, pp. 153-174, 2000.

[37] A. L. Mishev and J. N. Stamenov, "Present status and further possibilities for space weather studies at BEO Moussala," Journal of Atmospheric and Solar-Terrestrial Physics, vol. 70, no. 2-4, pp. 680-685, 2008.

[38] L. I. Miroshnichenko, "Solar cosmic rays in the system of solar-terrestrial relations," Journal of Atmospheric and SolarTerrestrial Physics, vol. 70, no. 2-4, pp. 450-466, 2008.

[39] A. L. Mishev, "A study of atmospheric processes based on neutron monitor data and Cherenkov counter measurements at high mountain altitude," Journal of Atmospheric and SolarTerrestrial Physics, vol. 72, no. 16, pp. 1195-1199, 2010. 

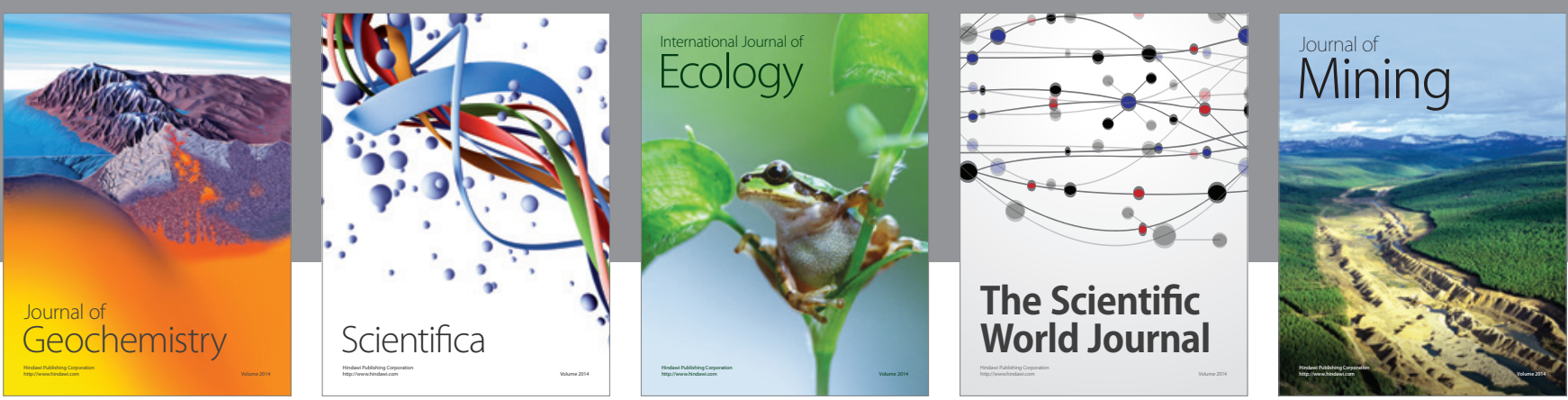

The Scientific World Journal
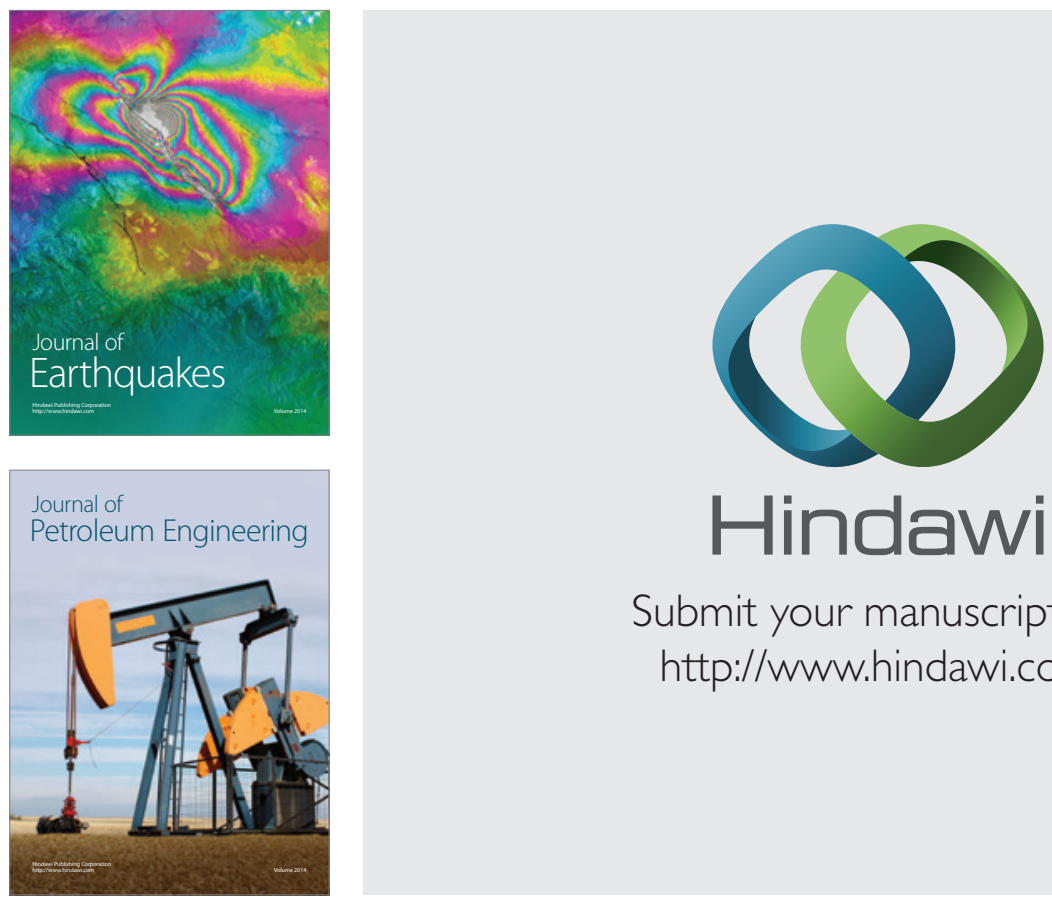

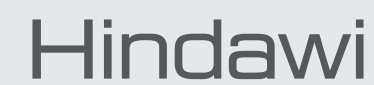

Submit your manuscripts at

http://www.hindawi.com
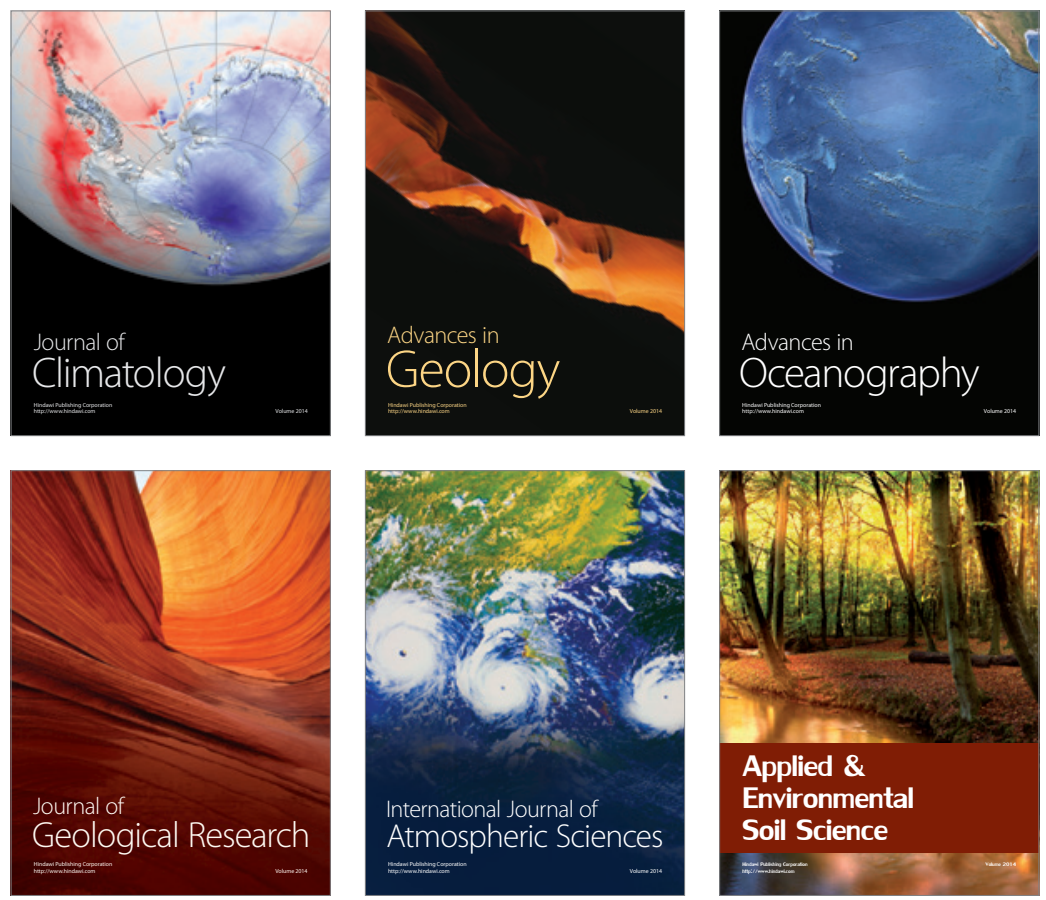
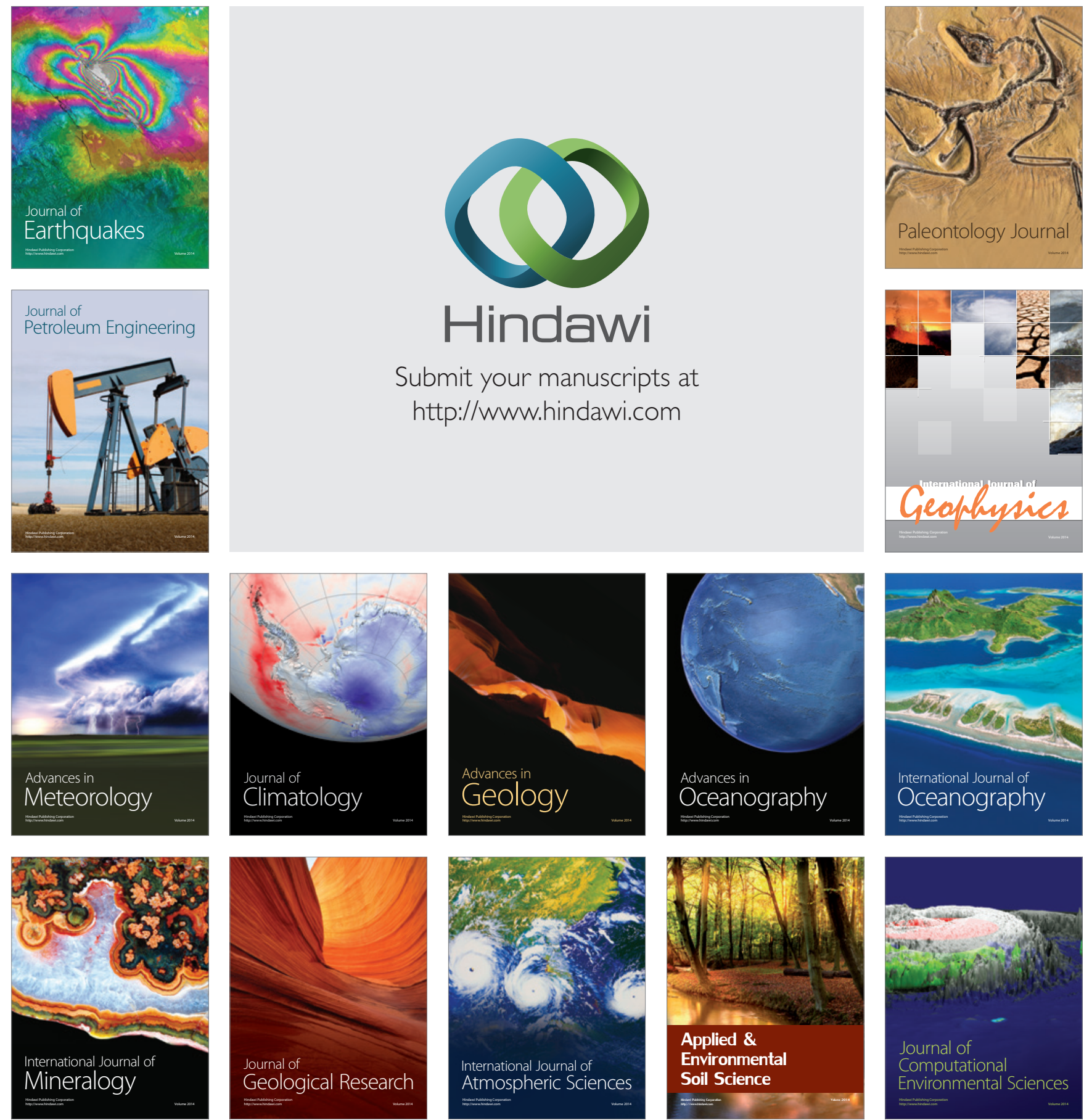